\title{
Introducing Engineering Concepts to Secondary Education through the Application of Pedagogical Scenarios in "Manuskills" Project.
}

\author{
Maria Margoudi ${ }^{1, *}$, Dimitris Kiritsis ${ }^{2}$ \\ ${ }^{1}$ École Polytechnique Fédérale de Lausanne (EPFL), Lausanne, Switzerland \\ maria.margoudi@epfl.ch \\ ${ }^{2}$ École Polytechnique Fédérale de Lausanne (EPFL), Lausanne, Switzerland \\ dimitris.kiritsis@epfl.ch
}

\begin{abstract}
The current paper identifies the lack of engineering concepts taught in secondary education and to this end proposes a new approach to be followed. The contents of the aforementioned approach are subject to the EU project Manuskills and the research conducted in its concept. The utilization of pedagogical scenarios is proposed as a tool for secondary school teachers towards the incorporation of engineering concepts into their teaching practice. The nature and the characteristics of pedagogical scenarios that constitute them appropriate for this purpose will be reported and analyzed.
\end{abstract}

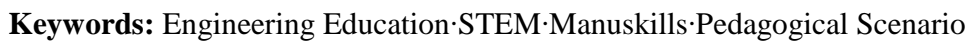

\section{Introduction}

The outcomes of this paper are subject to relevant research carried out in the concept of the EU project Manuskills. Manuskills' main aim is to raise awareness for specific manufacturing concepts, both in the secondary and higher education level. As a result of this attempt, a new need has emerged about introducing a new approach for the incorporation of engineering education to the current secondary education's program. In order to realize the objective of the project, a new approach was structured, leveraging the pre-existing concept of "pedagogical scenarios" and adapting it to the needs of engineering education. In the following chapters, the significance and the necessity of the pedagogical scenarios for the integration of engineering in secondary education will be argued.

\section{Theoretical Framework}

Following the advances in engineering education, it becomes evident that the first real reference to engineering related concepts for students doesn't occur until tertiary education. According to relevant researches, a large number of secondary school students fail in reaching math and science proficiency [1]. In order to support the broadening of

adfa, p. 1, 2011.

(C) Springer-Verlag Berlin Heidelberg 2011 
engineering within each one of the rest of the school subjects, the STEM (Science, Technology, Engineering and Mathematics) concept has been introduced. As mentioned by Gonzalez \& Kuenzi (2012) [2], STEM education refers to teaching and learning in the fields of science, technology, engineering, and mathematics. It typically includes educational activities across all grade levels. The importance of STEM education increases proportionately to the dependence of our society to technology. Consequently, it is vital that when students graduate from high school are comfortable with the STEM principles.

At this point, a special reference should be made to engineering education and the benefits it can provide for secondary education. First of all, engineering motivates students' learning of math and science concepts, which make technology possible. The engineering approach to learning, also enables the acquisition of knowledge irrelevant to STEM disciplines, like reading, writing, communication and design. The interdisciplinary nature of engineering, described above, occurs as a result of the kind of problems it addresses. Some of the learning gains of students, when engaging with engineering problems, are listed by Portsmore \& Rogers (2004) [3]. In more detail, students learn to: 1 . identify and formulate a problem, 2. design a solution, 3 . create and test a solution, 4. optimize and re-design and 5. communicate and disseminate the solution.

The review of the relevant literature presents an ambiguous picture. On the one hand, the fact of engineering education concepts providing an effective tool for students is generally accepted and recognized. On the other hand, engineering concepts are rarely addressed before university level education.

\section{Purpose and Vision}

The gap described in the theoretical framework, concerning the non-application of engineering concepts in secondary education, has several causes. Firstly, schools do not incorporate into their curricula a special subject for engineering. Even more importantly, very few teachers have knowledge of engineering concepts and therefore do not feel confident of including them in their teaching. Finally, a main cause for the low effectiveness and motivation regarding STEM education is the use of consolidated methods, techniques and tools by teachers.

In the current paper we suggest the use of pedagogical scenarios, as a means that will enable the incorporation and application of several different concepts of engineering education into secondary education.

The approach that will be described in the following chapters is developed under the EU funded project (FP7 609147) Manuskills.

\section{$4 \quad$ Manuskills Project}

Manuskills is a EU funded project (FP7 609147) which is oriented towards engineering education and specifically manufacturing. In more detail, Manuskills' main aim is to 
raise manufacturing awareness and even to develop the learner's understanding for specific manufacturing concepts, both in the secondary and higher education level [4].

The main target groups that the project is aiming at are teenagers and young adults, since, in general, they represent the most important beneficiaries of STEM and manufacturing education.

In order to achieve its goals, the project encompasses a series of experiments (available on Manuskills' Platform, http://demo.manuskills.org/). Each one of these experiments include the application of a delivery mechanism, which stands for cutting-edge ICT educational tools and the corresponding methods, so that the delivery of a course or even a concept, regarding STEM and/or manufacturing education, can be succeeded in a proper and contemporary frame. After following a searching and testing process, of both traditional and innovative tools, that are somehow linked to manufacturing education, the final bank consists of the following delivery mechanisms: Serious games, Simulations, Educational videos, Teaching Factory and Virtual Reality applications.

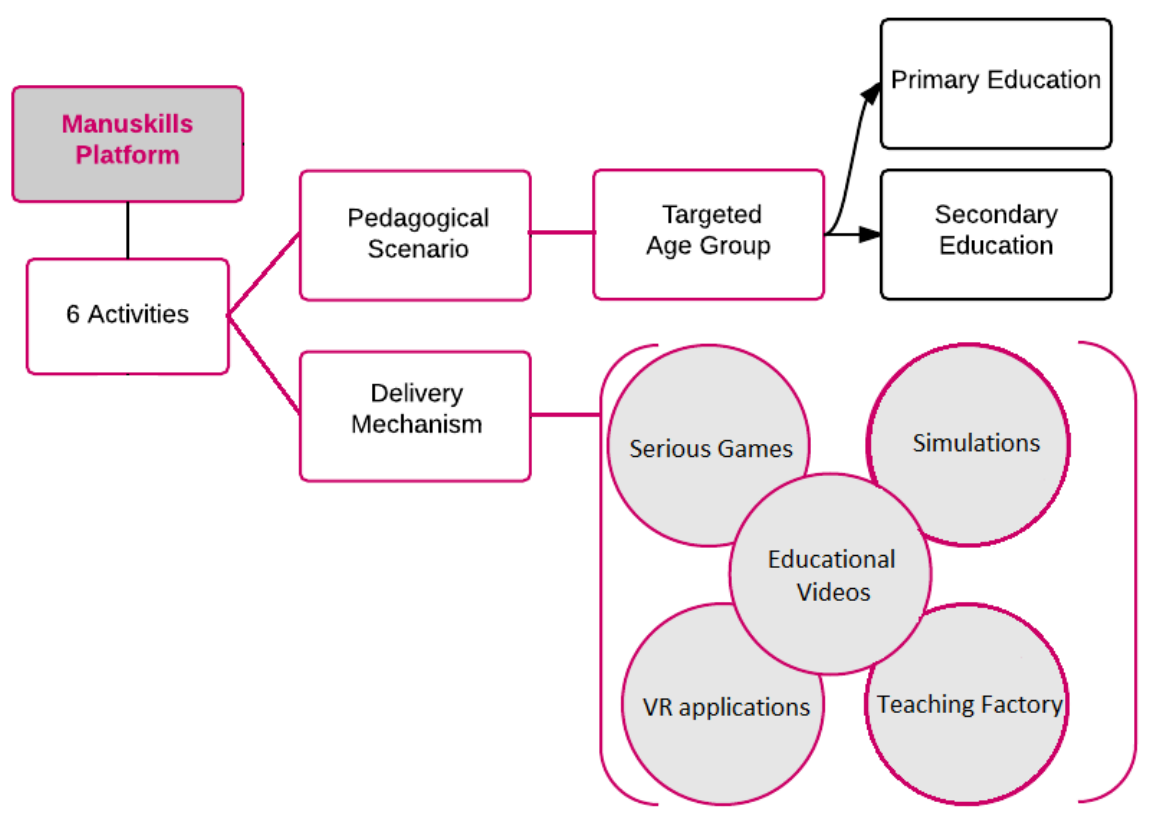

Fig. 1. Manuskills’ Approach

While preparing the application approach to secondary education, a new need arose. Since there is no specific school subject addressing engineering in general or especially manufacturing, a new way of incorporating our experiments to the existing secondary school's subjects was required. Moreover, another important aspect of the required solution was the fact that most secondary school teachers are not familiar with engineering concepts, and therefore feel intimidated about incorporating them into their teaching practice. 
Manuskills' answer to the aforementioned problem is the incorporation of pedagogical scenarios, to each one of the experiments, in order to introduce the aforementioned delivery mechanisms, to both secondary and tertiary education.

\section{$5 \quad$ Pedagogical Scenarios}

A pedagogical scenario -also referred to in literature as Educational Scenario or just Scenario- is a technology supported learning plan meant to be used by a teacher or any individual who is interested in incorporating technological features into a teaching procedure. As mentioned by Yiannoutsou \& Kynigo (2013) [5] the pedagogical scenarios are tools for teachers and describe the main elements of a plan of pedagogical activity, which employs the use of technology. Moreover, referring to the structure of their contents, they address aspects of the activity such as the epistemology of the domain, the social orchestration, the duration, the role and use of the tool. Despite all the aforementioned characteristics, the fact that makes a pedagogical scenario unique and that radically differentiates it from a simple learning plan, is the special reference made to the added educational value of the planned activities, in other words clarifying the nature of the innovation and the factors that determine its necessity [6].

For the needs of Manuskills project and towards the aim of a unified structure, the same template was used for all the pedagogical scenarios. The mentioned template consists of four interdependent modules, each of which contains several sections. The modules describe in detail the identity of the scenario, its application framework, the teaching-learning process to be followed and finally the implementation activities [7].

In the following paragraphs, we aim at giving a more detailed insight and a clearer view to the reader for each one of the described modules of the scenarios, as implemented and used for the purposes of the project. 


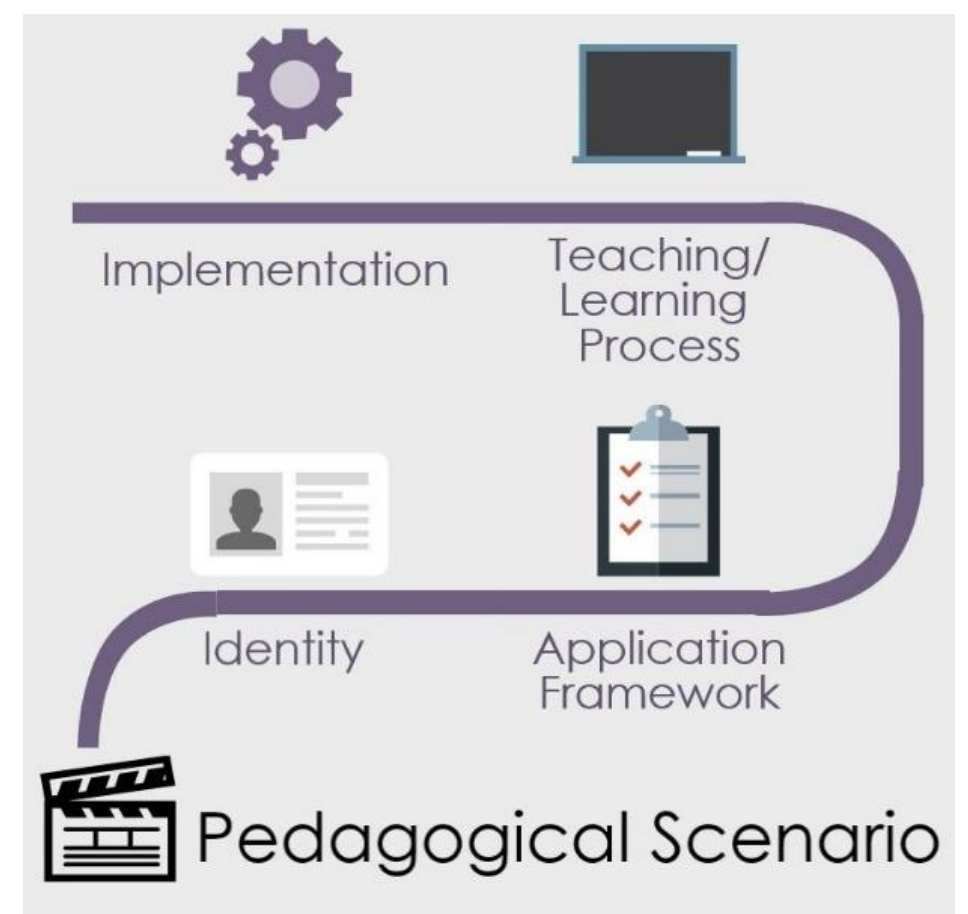

Fig. 2. Pedagogical Scenario's Structure

The first module provides a detailed view of the scenario's identity by clarifying several structure-related issues. Analytically, the reader receives details on the appropriate age group of students, as well as on the suggested school subjects to which the scenario could find application. Following these basic information, the cognitive domain that forms the scenario's background is briefly along with the scenario's learning objectives in terms of skills-to-be acquired by students. This module ends with a brief presentation of the topic and the rational of the scenario and also the added value of the activities that it encompasses.

The second module focuses on the application framework of the scenario and encompasses vital information for a teacher concerning the practical aspects of integrating the activity into a school's classroom. A special reference is made to the level and the pre-existing knowledge that the students are expected to possess, in order to engage with the scenario's activities. Also, the role that both the teacher and the students are expected to play, in terms of class orchestration, is described. All the aforementioned information of this module are topped with details concerning the time allocation of the activities and their duration, as well as details on the software applications and educational materials needed for the attainment of the planned activities.

The next module of the pedagogical scenario is referring to the teaching and learning process that is expected to take place. It is the most theoretical of all the modules and it basically constitutes necessary theoretical background of the scenario. It includes details on the cognitive terms used and the pedagogical framework that it encompasses in 
its activities. Finally, the teaching methodology and accompanying strategies are listed and briefly explained.

The fourth and final module is also considered to be the most important one. The subject of this module are the actual activities that constitute the pedagogical scenario. All the activities are listed and described in a chronologically structures way. For this purpose, the whole procedure is divided into phases and for each phase practical information are provided, like its duration, teacher's and students' role etc.

In order to enable the reader form a more clear view on the scenario's structure, which was employed for the needs of Manuskills [7], all the modules and their contents are analytically listed below:

1. Scenario Identity
(a) Creators
(b) Subject Matter
(c) Topic of Scenario
(d) Rational of Scenario
(e) Added Value

2. Scenario Application Framework
(a) Level \& Pre-existing Knowledge of the Students
(b) Class Orchestration
(c) Social Orchestrator
(d) Time Allocation \& Duration
(e) Software Application \& Materials

3. Teaching-Learning Process
(a) Theoretical Framework
(b) Pedagogical Framework-Model
(c) Methodological Framework-Model
(d) Goals-Expected Results

4. Scenario Implementation
(a) Learning Activities
(b) Worksheets
(c) Enrichment of Scenario

\section{Added Value for Engineering Education}

The first module of the pedagogical scenario's structure concerns the "Scenario Identity" and it contains information about the target group of the scenario, such as the age of students and their learning level. For engineering education this translates into engineering content adapted appropriately to the needs of each age and learning level. Another section of the same module is dedicated to the corresponding school subject or learning domain, in general. This section aims at incorporating various engineering concepts into already existing school subjects (such as Math, Science etc.). Also, particular emphasis is placed on the correspondence to each country's official curriculum. 
Finally, an important section refers to the added value of each particular scenario, explaining the profits gained from the use of technology for the optimal presentation and explanation of engineering concepts.

The second module of the pedagogical scenario is revolving around its "Application Framework". The sections of this module covers a wide range of information, including the level of pre-existing knowledge, the suggested classroom organization, time allocation and duration of the activities and details about the leveraged software or any other appropriate materials. All the aforementioned information constitute a useful and necessary tool in the hands of a teacher, in order to organize his/her teaching practice.

Another important module is the "Teaching - Learning Process", which consists of the necessary frameworks that eventually structure the theoretical, pedagogical and methodological background of the learning activities. Firstly, the section about the theoretical framework elaborates on basic engineering concepts related to the subject or the problem in question, providing all the necessary scientific references for the teacher to explore. The pedagogical and methodological frameworks refer to the organization of the teaching and learning, by providing state of the art didactic models and the latest teaching approaches to be followed. In support of the adopted theories, scientific references are available for the teacher.

The final module of a pedagogical scenario concerns its "Implementation". This module includes the most "practical" sections of a scenario. In more detail, sections referring to learning activities and worksheets can be found in this module, providing information and guidance, not only for the students involved, but also for the teachers.

\section{Conclusions}

At the beginning of this paper a knowledge gap was identified in the existing literature, concerning engineering education. In more detail, it is evident that even within STEM education, concepts that revolve around engineering do not find easily application into secondary education. The causes of the aforementioned situation cab be divided into three general categories, accordingly, there are school-related, teacher-related and tools-related causes.

School-related causes are linked to the fact that there is no particular school subject on engineering below the university education level. This situation makes it difficult for the teachers to identify a school subject that could be relevant for a particular engineering concept. The detailed description of a pedagogical scenario, of the previous chapter, makes clear its contribution to this particular issue by clarifying, each time, the appropriate school subject and learning domain for the taught engineering concept ("Scenario Identity" module).

The second cause relates to teachers themselves, as it is a fact that most of them are not familiar with engineering concepts and therefore do not feel confident in incorporating them into their teaching practice. In order to tackle this issue, the pedagogical scenario incorporates into its structure a variety of information about the basic concepts of the engineering subject in question each time ("Theoretical Framework" section). 
Moreover, many information about the teaching procedure in general are available ("Scenario Application Framework" module).

Finally, the third cause of the low inclusion of engineering related concepts in STEM education is closely linked to the use of consolidated tools by the teachers. A pedagogical scenario's structure is oriented towards providing appropriate methods, techniques and even technological tools' suggestions for teachers, towards incorporating engineering in their teaching practice ("Pedagogical Framework" and "Methodological Framework" sections).

Concluding, we argue that the pedagogical scenario approach is capable of incorporating all the necessary components to tackle the main causes that result to the low representation of engineering in STEM education and therefore enable the introduction of engineering concepts to even younger ages of students.

Acknowledgements. The research conducted in the frame of "Manuskills", leading to these results has received funding from the European Community's Seventh Framework Programme (FP7/2007-2013) under grant agreement nº 609147.

\section{$8 \quad$ References}

1. Kuenzi, J. J. (2008). Science, technology, engineering, and mathematics (stem) education: Background, federal policy, and legislative action.

2. Gonzalez, H. B., \& Kuenzi, J. J. (2012, August). Science, technology, engineering, and mathematics (STEM) education: A primer. Congressional Research Service, Library of Congress.

3. Portsmore, M. D., \& Rogers, C. (2004). Bringing engineering to elementary school. Journal of STEM education, 5.

4. Perini, S., Oliveira, M., Costa, J., Kiritsis, D., Hansen, P. H. K., Rentzos, L., ... \& Taisch, M. (2014). Attracting Young Talents to Manufacturing: A Holistic Approach. In Advances in Production Management Systems. Innovative and Knowledge-Based Production Management in a Global-Local World (pp. 626-633). Springer Berlin Heidelberg.

5. Yiannoutsou, N., \& Kynigo, C. (2013). Boundary objects in educational design research: Designing an intervention for learning how to learn in collectives with technologies that support collaboration and exploratory learning. In T. Plomp, \& N. Nieveen (Eds.), Educational design research - Part B: Illustrative cases (pp. 357-379). Enschede, the Netherlands: SLO.

6. Kynigos, C., \& Kalogeria, E. (2012). Boundary crossing through in-service online mathematics teacher education: the case of scenarios and half-baked microworlds. ZDM, 44(6), 733-745.

7. Fragkaki, M., (2008). Structure of Learning Scenarios. EduTubePlus - A European Curriculum Related Video Library and Hybrid e-services for the Pedagogical Exploitation of Video in Class. Deliverable WP6 Pedagogical Framework-Pilot Implementation/T61 Pedagogical Framework. Retrieved from http://www.academia.edu/5170480/Pedagogical_Scenario_Structure . 